Article

\title{
Optimal Charging and Discharging Scheduling for Electric Vehicles in a Parking Station with Photovoltaic System and Energy Storage System
}

\author{
Leehter Yao *, Zolboo Damiran and Wei Hong Lim \\ Department of Electrical Engineering, National Taipei University of Technology, Taipei 10608, Taiwan; \\ t104319007@ntut.edu.tw (Z.D.); whlim@ntut.edu.tw (W.H.L.) \\ * Correspondence: 1tyao@ntut.edu.tw; Tel.: +886-2-771-2171 (ext. 2174) \\ Academic Editor: Chunhua Liu \\ Received: 20 February 2017; Accepted: 13 April 2017; Published: 17 April 2017
}

\begin{abstract}
The economic and environmental benefits brought by electric vehicles (EVs) cannot be fully delivered unless these vehicles are fully or partially charged by renewable energy sources (RES) such as photovoltaic system (PVS). Nevertheless, the EV charging management problem of a parking station integrated with RES is challenging due to the uncertain nature of local RES generation. This paper aims to address these difficulties by deploying an energy storage system (ESS) in parking stations and exploiting the charging and discharging scheduling of EVs to achieve better utilization of intermittent PVS for EV charging. A real-time charging optimization scheme is also formulated, using mixed-integer linear programming (MILP) to coordinate the charging or discharging power of EVs along with the power dispatches of power grid and ESS based on the vehicles' charging or discharging priorities and electricity price preferences. Extensive simulations show that the proposed approach not only maximizes the satisfaction of EV owners in terms of fulfilling all charging and discharging requests, but also minimizes the overall operational cost of the parking station by prioritizing the utilization of energy from PVS, ESS, and scheduling of every EV's charging and discharging.
\end{abstract}

Keywords: energy storage system; electric vehicle; mixed-integer linear programming (MILP); photovoltaic system; renewable energy source

\section{Introduction}

The depletion of fossil fuels and increasing greenhouse gas emissions associated with their wide usage, especially in the transportation sector, have received growing attention from the public in recent decades. Extensive use of electric vehicles (EVs) instead of traditional internal combustion engine vehicles in the transportation sector is envisioned as one promising solution to mitigate the crisis, motivated by the lower operational costs and lower gas emissions of EVs. Nevertheless, the integration of large-scale EVs into existing power system is challenging due to the massive electricity demands of these emerging loads. Uncoordinated charging of large EV fleets in venues such as parking stations tends to impose significant risk on the power system in terms of transmission and/or distribution system overloading, power losses, and voltage deviations [1].

To alleviate the technical drawbacks created by the high penetration level of EVs in the power system, extensive research was conducted by leveraging the controllable charging rate and flexible load characteristic of EVs. Numerous coordinated charging schemes were proposed in the literature aiming to level, delay, and regulate large-scale EV charging without compromising the stability of the power system [2-4]. A proper EV charging coordination scheme can benefit the utility further by providing demand response or ancillary services in the case of a system emergency, as these aggregated 
charging loads create an enormous demand with a notable impact on power grids [5-7]. Most of these previous works share a common pitfall by considering the electricity used for EV charging as supplied from the grid. The main goal of introducing EVs as a solution to reduce air pollution is therefore diminished because the carbon footprint produced from the transportation sector is shifted to the power generation sector. Some state-of-the-art studies [8,9] advocated the necessity of integrating renewable energy sources (RESs) and EVs into a smart grid in order to maximize the environmental and economic benefits of EVs. Therefore, deploying RES such as a photovoltaic system (PVS) as a complete or partial power source for EV charging in a parking station is expected to further reduce greenhouse gas emissions [10]. Nevertheless, the optimal energy management of an EV parking station integrated with PVS is challenging due to the high inter-temporal variation of electricity production from PVS and variable EV traffic in the parking station. Significant research efforts are needed to explore suitable solutions for overcoming these technical challenges before the integration of PVS in an EV parking station becomes commonplace.

This paper aims to provide insight to address the aforementioned challenges by incorporating an energy storage system (ESS) in the EV parking station supplied by a conventional power grid and PVS. ESS offers some desirable characteristics of controllable charging and discharging rates that enable it to be leveraged by the charging management system (CMS) to better utilize the intermittent PVS and steady power grid for EV charging throughout the scheduling process. Particularly, ESS can be charged by the excess PVS production or by the power grid during off-peak pricing periods, while the energy stored in ESS can be utilized for EV charging when the electricity price rises. The vehicle-to-grid (V2G) capability of EVs is also exploited in this study, given that the energy stored in EV batteries can be accumulated or released at different intervals of their stay, depending on the state of charge (SOC), electricity prices, and power of PVS. However, searching for the optimal utilization of both ESS and EVs as energy storage in a parking station integrated with PVS and a power grid is challenging due to the additional complexity brought by these new entities. A sophisticated EV charging optimization scheme is proposed in this paper, optimizing the charging and discharging scheduling of EVs and ESS in real time with CMS. Apart from preventing the parking station from overloading, the proposed work also aims to minimize the operational costs of the parking station by prioritizing the use of energy obtained from PVS, ESS, and the power grid for EV charging. The technical novelty and main contributions of this paper are summarized as follows:

1. To the best of the authors' knowledge, the work proposed in this paper is the first to exploit the benefits of both the ESS and V2G capability of EVs to better utilize the intermittent PVS for EV charging in a parking station.

2. A real-time charging scheduling scheme is proposed to coordinate the charging or discharging of EVs along with dynamic electricity prices, ESS, and PVS.

3. An EV charging optimization problem is formulated using mixed integer linear programming (MILP), aiming to maximize the satisfaction of EV owners in terms of simultaneously fulfilling all charging requests and minimizing the overall operational costs of the parking station.

The remaining of this paper is organized as follows. Section 2 presents the related work. The system model of an EV parking station integrated with RES and ESS is described in Section 3, followed by the MILP formulation used to solve the EV charging scheduling problem in Section 4 . Extensive computer simulations are presented in Section 5, and conclusions are drawn in Section 6.

\section{Review of Existing Literature}

Substantial works were proposed in recent years to address the EV charging scheduling problem mentioned in [2-4]. Linear programming (LP) is one approach commonly used to pursue EV charging scheduling. An LP model was formulated in [11] to maximize the profit of aggregator and minimize the charging cost of EVs. In [12], a hierarchical control scheme was developed to minimize the charging cost and peak load caused by EV charging across multiple aggregators. The overall charging profile of each 
aggregator is first allocated at the power distribution level and a heuristic method is then used to obtain the charging rate of each EV. The aggregator's profit in $[7,13]$ is maximized by MILP and fuzzy linear programming (FLP), respectively. Two LP models were formulated in [14] to optimize EV charging in a parking station with different points of view, i.e., to maximize either the operator's revenue or the number of completely charged EVs. Two contradictory objectives-minimizing the charging cost of an EV parking station and maximizing EV users' satisfaction-were simultaneously achieved in [15] via LP and a modified convex relaxation scheme. Other optimization techniques such as quadratic programming (QP) [16,17], dynamic programming (DP) [18], swarm intelligence [19-21], and game theory [22] were also used for EV charging scheduling. Different objective functions including the minimization of EV charging cost and grid's load variance, maximization of the aggregator's profit, average state-of-charge (SOC) of EVs, and EV owners' utilization rate in the parking station, were utilized in these works.

Some recent studies suggested the crucial role of RES such as PVS or wind energy for EV charging in order to fully deliver its environmental and economic values, leading to the growing attention of optimal energy management issues of an EV parking station integrated with RES. An online multi-objective framework was developed in [23] for an energy hub from the perspective of both EV users and system operator, aiming to minimize distribution system losses and total rescheduling costs and to maximize the wind energy utilization for EV charging. In [24], the V2G regulation of EVs was optimized using an LP-based hierarchical framework to minimize the operational costs of a distribution grid with intermittent wind power. Lyapunov optimization was used in [25] to minimize EV charging costs and waiting time for fulfilling EV charging requests without requiring prior knowledge of PVS generation and EV charging demands. In [26], a two-stage mechanism was formulated using QP to tackle the EV charging involving PVS. The minimization of charging costs and peak to average (PAR) of the system were attained by first solving the offline optimal energy dispatch problem, followed by the dynamic charging coordination of EV. A classification scheme was suggested in [27] to categorize the EVs in a solar-powered parking station based on their charging behavior. An MILP energy trading scheme was then proposed to minimize the charging cost by encouraging more active participation of EVs in V2G. In [28], a fuzzy controller was designed to minimize the charging cost of an EV parking station consisting of PVS by adjusting the charging rates of EVs in real time based on their respective power requirements and electricity tariff.

While there has been growing interest in exploring the potential of PVS or wind energy as a complete or partial source for an EV parking station, limited efforts have been made to address the intermittency of PVS or wind energy availability and the uncertainty of EV charging demands in a parking station. One possible solution to overcome these drawbacks involves the integration of ESS into these intermitted power sources, leading to the deployment of various hybrid energy systems (HESs) in different smart grid applications [29]. Nevertheless, ESS tends to generate high operational costs and strong operational constraints. The power flow management between different entities of HES needs to be addressed carefully in order to reduce the operation costs by optimizing the utilization of ESS and renewable generation, as well as matching local production with local consumption. For instance, a lithium-ion battery and fuel cell are used in [30] to prevent the power failure of a DC microgrid consisting of PVS and wind energy, in which a fuzzy logic controller (FLC) was designed to improve the battery's life by maintaining its desired SOC. In [31], a similar HES was deployed and the operation of ESS was controlled using a multi-agent FLC by referring to the SOC of both the DC bus and ESS. Another energy management scheme used to minimize the grid power fluctuations or consumption of a residential grid-connected microgrid while keeping the SOC of ESS within secure limits was reported in [32-34]. The optimal operation of a grid-connected HES consisting of PVS, fuel cell, and ESS was formulated in [35], using MILP to minimize either the running costs or the $\mathrm{CO}_{2}$ emissions. In [36], the electricity cost of a grid-connected smart home consisting of PVS, ESS, and EVs was minimized using MILP based on the dynamic electricity prices. The optimal power flow management problem of a grid-connected PVS with ESS in [37] was solved using DP to minimize the 
cost of peak shaving by considering the battery ageing effect in the optimization. In [38], the charging and discharging of ESS in similar HES were controlled using convex optimization to maximize cost savings by considering the energy loss of ESS due to the rate capacity effect. A central controller was designed in [39] to compensate for the power fluctuation caused by the renewable generation in the microgrid by coordinating the operation of heat pumps and ESS modeled as a two-tank energy storage device. Most existing works [30-36] related to the life cycle of ESS with its SOC assumed that no significant maintenance costs were incurred by ESS as long as ESS was not overcharged or over-discharged beyond the limits of SOC. Meanwhile, some limited studies [37-39] that considered the ESS models with more comprehensive degradation effects during optimization tend to employ a nonlinear mathematical formulation, which is generally not feasible for real-time applications.

The proposed EV charging scheme is compared with previous works of EV charging optimization in Table 1 in terms of the objective model, solution method, and the presence of RES, ESS, and V2G. The difference between the proposed approach and those in the literature are summarized as follows.

1. The works in $[7,11,12,14-22]$ rely on the electricity generated from conventional units for EV charging. The proposed work considers PVS as a power source for EV charging, hence is designed to fully deliver the economic and environmental advantages of EVs.

2. Only the V2G capability of EVs was exploited to mitigate the intermittent RES in $[24,27,28]$, while the proposed approach harnesses both the energy storage capability of ESS and the V2G capability of EVs to better utilize the PVS for EV charging.

3. Some previous works aimed to maximize the satisfaction of EV users by completing all charging demands (e.g., [19-21]) or minimizing the operational costs (e.g., [16,24,27,28]). A simple yet efficient objective function is proposed to attain these contradictory goals by considering (a) the charging or discharging priorities of EVs and (b) the preferred electricity price as the coefficient in an objective function to dictate the power dispatches of the power grid and ESS.

Table 1. Comparisons of the proposed work with other EV charging schemes.

\begin{tabular}{|c|c|c|c|c|c|}
\hline Reference & Objective Model & Solution Method & RES & V2G & ESS \\
\hline Jin et al. [11] & Max. aggregator's profit, Min. charging cost & LP & No & No & No \\
\hline Xu et al. [12] & Min. electricity cost, Min. system peak load & LP & No & No & No \\
\hline Jin et al. [13] & Max. aggregator's revenue & MILP & No & No & Yes \\
\hline Ansari et al. [7] & Max. aggregator's profit & FLP & No & No & No \\
\hline Kuran et al. [14] & Max. revenue or Max. EV users' satisfaction & LP & No & No & No \\
\hline Yao et al. [15] & Max. charged EV number, Min. charging cost & $\begin{array}{l}\mathrm{LP}+\text { convex } \\
\text { relaxation }\end{array}$ & No & No & No \\
\hline He et al. [16] & Min. charging cost & $\mathrm{QP}$ & No & Yes & No \\
\hline Jian et al. [17] & Min. overall load variance & $\widehat{Q P}$ & No & Yes & No \\
\hline Han et al. [18] & Max. aggregator's profit & $\mathrm{DP}$ & No & Yes & No \\
\hline Su et al. [19-21] & Max. average SOC of all EVs for next time step & Swarm intelligence & No & No & No \\
\hline Zhang and Li [22] & Max. EV user utilization & Game theoretic & No & No & No \\
\hline Moeini-Aghtaie et al. [23] & $\begin{array}{l}\text { Min. system losses, Min. rescheduling cost, } \\
\text { Max. RES utilization for EV charging }\end{array}$ & $\begin{array}{l}\text { Muti-objective } \\
\text { optimization }\end{array}$ & Wind & No & No \\
\hline Gao et al. [24] & Min. operational cost & LP & Wind & Yes & No \\
\hline Jin et al. [25] & $\begin{array}{l}\text { Min. charging cost, Min. waiting time of } \\
\text { fulfilling EV charging request }\end{array}$ & $\begin{array}{l}\text { Lyapunov } \\
\text { optimization }\end{array}$ & PVS & No & No \\
\hline Wang et al. [26] & Min. charging cost, Min. PAR of system & $\mathrm{QP}$ & PVS & No & No \\
\hline Tushar et al. [27] & Min. charging cost & MILP & PVS & Yes & No \\
\hline Mohamed et al. [28] & Min. charging cost & Fuzzy logic & PVS & Yes & No \\
\hline Proposed work & $\begin{array}{l}\text { Max. satisfaction of EV owners, Min. } \\
\text { operational cost of parking station }\end{array}$ & MILP & PVS & Yes & Yes \\
\hline
\end{tabular}

\section{EV Charging Management in the Proposed Parking Station}

Consider a parking station consisting of a number of charging poles for EV charging and discharging under a day-ahead electricity tariff environment. The parking station is equipped with a PVS that serves as an alternative energy source to charge the connected EVs. The parking station is also connected to a power grid, allowing EV charging using conventional power from utility and 
EV discharging to power grid. Both the energy storage capability of ESS and the V2G capability of EVs are further exploited in the parking station to reduce electricity consumption from the power grid. This goal can be achieved by charging both ESS and EVs with PVS as much as possible, and the stored energy in both ESS and EV is discharged to the grid. The parking station operator can also leverage the variations of electricity prices to reduce the operational costs by selecting proper time slots to purchase electricity from the grid and sell excessive energy to the grid if the electricity prices are right.

Given that each EV has a different arrival time, departure time, and residual battery capacity, an optimal EV charging and discharging scheduling is required to determine the charging and discharging priorities of EVs to ensure charging and discharging fairness, as well as the requirement of supplying adequate energy for every EV before departure. Moreover, the simultaneous charging or discharging of all connected EVs and ESS are prohibited in a parking station because of the installation capacity limit assigned to the electric distribution system. Nevertheless, these charging and discharging operations of connected EVs and ESS can be leveled, delayed, and managed intelligently.

A CMS is designed to optimize the charging and discharging scheduling of EVs in real time and to optimize the power dispatch from the power grid, ESS, and PVS with the dynamic electricity tariff. As illustrated in Figure 1, the day-ahead electricity price signals are sent from the utility company to the CMS via the Internet. A smart meter is installed in the parking station to record the electricity to or from the grid. This recorded power profile is then returned to the utility through the Internet. It is assumed that each charging pole can record the EV's arrival time and allow every EV driver to input their estimated departure time. Two-way communication between the CMS and other entities in the parking station are established with a local area network (LAN) to enable the CMS to monitor the solar power generated from PVS, the residual energy of ESS, and the connection status of each charging pole.

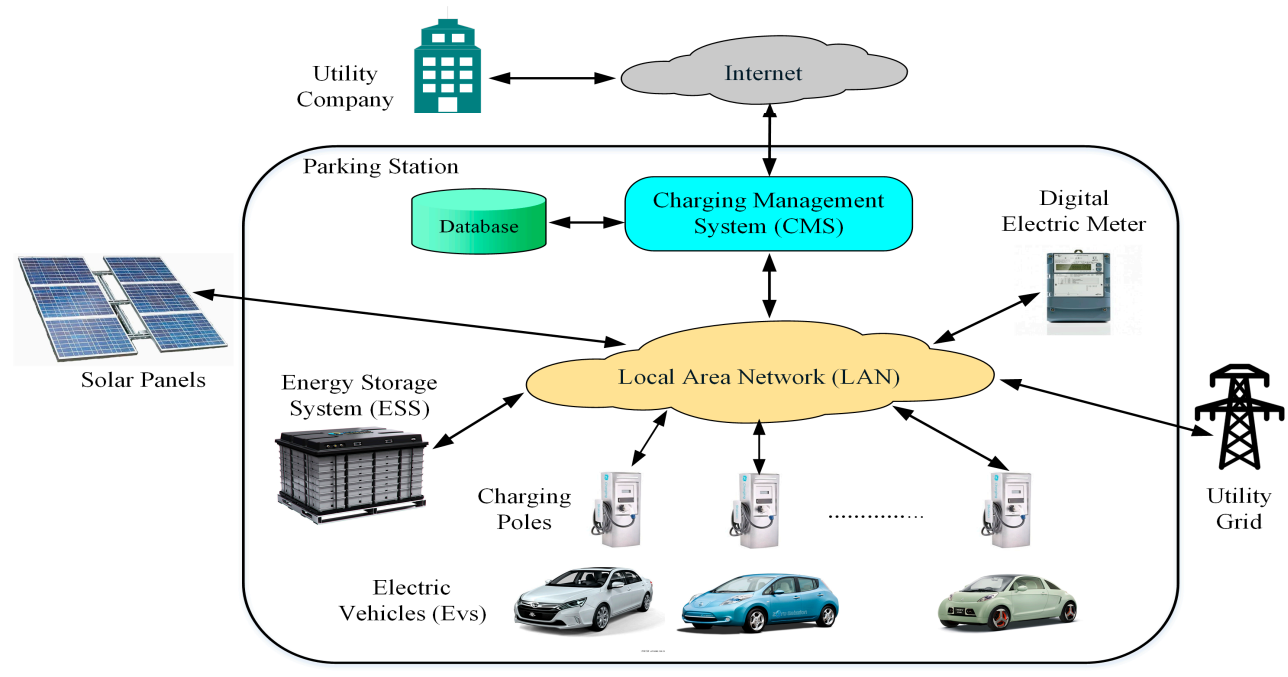

Figure 1. Illustration of EV charging and discharging in a parking station with ESS, PVS, and the power grid.

\section{Optimal Charging Scheduling}

Assume that the parking station consists of $N$ charging poles to charge the incoming EVs. An online optimization scheme is developed in CMS, not only to coordinate the charging and discharging scheduling of these $N$ charging poles, but also to determine the optimal power dispatch to/from the power grid, ESS, and PVS. The EV charging scheduling problem herein is approximated with the slotted time modeling approach, i.e., the total available time period $H$ for charging during a day is uniformly divided by a sampling interval $T_{s}$ into $J$ intervals, where $J=H / T_{s}$. Suppose that the $n$-th charging pole is connected by an EV that arrives at a real arrival time of $t_{n}^{\text {arr }}, n=1, \ldots, N$, the 
charging pole is activated by initializing its sign-on status. A ceiling rounding operator $\lceil\cdot\rceil$ is used to obtain the associated arrival time step $\kappa_{n}^{\text {arr }}$ by mapping the real value ratio of $t_{n}^{\text {arr }} / T_{s}$ onto the smallest following integer, i.e., $\kappa_{n}^{\text {arr }}=\left\lceil t_{n}^{a r r} / T_{s}\right\rceil$. Let $t_{n}^{d e p}$ be the estimated departure time provided by EV driver to the $n$-th charging pole. A floor rounding operator $\lfloor\cdot\rfloor$ is applied to acquire the corresponding departure time step denoted as $\kappa_{n}^{d e p}$ by mapping the real value ratio of $t_{n}^{d e p} / T_{s}$ as the largest previous integer, where $\kappa_{n}^{d e p}=\left\lfloor t_{n}^{d e p} / T_{s}\right\rfloor$.

Suppose that $\delta_{n}^{j}$ is a binary parameter used to indicate the connect status of each $n$-th charging pole at the $j$-th time step for $n=1, \ldots, N$ and $j=1, \ldots, J$. In particular,

$$
\delta_{n}^{j}= \begin{cases}1, & \forall j \in\left[\kappa_{n}^{a r r}, \kappa_{n}^{d e p}\right] \\ 0, & \forall j \in[1, J] \backslash\left[\kappa_{n}^{a r r}, \kappa_{n}^{d e p}\right],\end{cases}
$$

implying that $\delta_{n}^{j}$ is set to be 1 if the $n$-th charging pole is occupied and $\delta_{n}^{j}=0$, otherwise. Let $P_{n}^{c, m a x}$ be the maximum charging power provided to EV connected to the $n$-th charging pole and $P_{n}^{d, m a x}$ be the maximum discharging power drawn from this EV. Then, two continuous variables known as $s_{n}^{c, j} \in[0,1]$ and $s_{n}^{d, j} \in[0,1]$ are used to represent the charging and discharging rates of the $n$-th charging pole, respectively. Therefore,

$$
\begin{gathered}
s_{n}^{c, j} \leq \delta_{n}^{j} \\
s_{n}^{d, j} \leq \delta_{n}^{j} .
\end{gathered}
$$

The proposed approach aims to optimize the charging schedule by simultaneously maximizing EV owners' satisfaction in fulfilling all charging requests and minimizing the operational costs of the parking station by considering the dynamic electricity tariff, the solar power generated from PVS, and the energy stored in ESS. To achieve these objectives, two criteria, (a) EV charging and discharging priorities; as well as (b) the preferred electricity price to perform the power dispatches of power grid and ESS, are considered to determine the optimal charging or discharging rate of all charging poles at each time step.

\subsection{EV Charging and Discharging Priorities}

Two weightings are utilized to denote the charging and discharging priorities of each $n$-th connected EVs at different time steps by considering both the capacity to refill the EV's battery and the remaining charging time. Define $D_{n}^{j}$ as the remaining charging time steps of the EV connected to the $n$-th charging pole at the $j$-th time step, then

$$
D_{n}^{j}=\kappa_{n}^{d e p}-j
$$

Let $\gamma_{n}^{\max }, \gamma_{n}^{j}$, and $E_{n}^{\text {cap }}$ be the upper limit of SOC, current SOC level, and battery capacity of EV connected to the $n$-th charging pole, respectively. Then, the weighting of charging and discharging priorities for every $n$-th connected EV at any $j$-th time step can be represented as $w_{n}^{c, j}$ and $w_{n}^{d, j}$ as follows:

$$
\begin{aligned}
& w_{n}^{c, j}=\left\{\begin{array}{ll}
\frac{E_{n}^{c a p}\left(\gamma_{n}^{\max }-\gamma_{n}^{j}\right)}{P_{n}^{c, m a x} D_{n}^{j}} & , \text { if } \delta_{n}^{j}=1 \\
0 & , \text { if } \delta_{n}^{j}=0
\end{array},\right. \\
& w_{n}^{d, j}=\left\{\begin{array}{ll}
\frac{P_{n}^{d, m a x} D_{n}^{j}}{E_{n}^{c a p}\left(\gamma_{n}^{\max }-\gamma_{n}^{j}\right)} & , \text { if } \delta_{n}^{j}=1 \\
0 & , \text { if } \delta_{n}^{j}=0
\end{array} .\right.
\end{aligned}
$$


Both the numerator in Equation (5) and the denominator in Equation (6) represent the remaining capacity required to meet the battery's upper limit $\gamma_{n}^{\max }$. Both Equations (5) and (6) imply that EVs with less SOC have more urgent charging needs, while those with higher SOC are suitable for V2G operations. Each of the $n$-th charging poles is controlled to charge or discharge the connected EV without exceeding the maximum power of $P_{n}^{c, m a x}$ and $P_{n}^{d, m a x}$, respectively. Therefore, the denominator of Equation (5) and the numerator of Equation (6) imply the maximum energy that can be provided to and supplied by the EVs. EVs with shorter $D_{n}^{j}$ have more urgent charging needs and need to be charged as much as possible prior to their departure, whereas those with longer $D_{n}^{j}$ are ready to discharge and sell the stored energy to the grid. Both of $w_{n}^{c, j}$ and $w_{n}^{d, j}$ are normalized into $[0,1]$. Notably, when a connected EV is unplugged from the $n$-th charging pole and departs the parking station, this charging pole is signed off by resetting both the weighting $w_{n}^{c, j}$ and $w_{n}^{d, j}$, as well as the connect status $\delta_{n}^{j}$ to 0 for $n=1, \ldots, N$ and $j=1, \ldots, J$. The charging pole is activated again when a new EV connects with it at later time steps and the associated weighting $w_{n}^{c, j}$ and $w_{n}^{d, j}$ are recomputed using Equations (5) and (6), respectively.

\subsection{Preference on Electricity Prices for Power Dispatches of Power Grid and ESS}

Apart from fulfilling all charging requests of connected EVs, the parking station operator also tends to leverage the time-varying electricity price to minimize the operational costs, as driven by the economic motivation. In general, more EVs should be scheduled for charging during the off-peak electricity tariffs, while the discharging of EVs is encouraged at time periods when the electricity price becomes expensive. Suppose that $\alpha_{\max }$ and $\alpha_{\min }$ refer to the maximum and minimum values of day-ahead electricity prices, respectively. Two auxiliary parameters known as $\rho_{n}^{c, j}$ and $\rho_{n}^{d, j}$ are then used to quantify the preference levels of the $n$-th charging pole to charge and discharge the connected $\mathrm{EV}$, respectively, at the $j$-th time step given the electricity price of $\alpha^{j}$. Therefore,

$$
\begin{gathered}
\rho_{n}^{c, j}=\left\{\begin{array}{ll}
\frac{\left(\alpha_{\max }-\alpha^{j}\right)}{\left(\alpha_{\max }-\alpha_{\min }\right)} & , \text { if } \delta_{n}^{j}=1 \\
0 & \text {,if } \delta_{n}^{j}=0
\end{array},\right. \\
\rho_{n}^{d_{, j}}=\left\{\begin{array}{ll}
\frac{\left(\alpha^{j}-\alpha_{\min }\right)}{\left(\alpha_{\max }-\alpha_{\min }\right)} & , \text { if } \delta_{n}^{j}=1 \\
0 & , \text { if } \delta_{n}^{j}=0
\end{array} .\right.
\end{gathered}
$$

From Equation (7), a larger value preference term $\rho_{n}^{c, j}$ is obtained for the lower electricity tariff $\alpha^{j}$ and vice versa. The opposite preference is designed in $\rho_{n}^{d_{j} j}$, as in Equation (8), in response to the variation in electricity price. Both $\rho_{n}^{c, j}$ and $\rho_{n}^{d, j}$ are normalized into $[0,1]$ by the terms in the denominators of Equations (7) and (8), respectively.

To further minimize the operational costs, the parking station operator also needs to consider the preference on electricity price in the power dispatches of power grid and ESS at every time slot. Intuitively, the parking station operator tends to discharge the ESS and sell as much electricity as possible to the power grid during time periods with a higher electricity price in order to generate higher revenues and vice versa. The power dispatches for both ESS and the power grid at every $j$-th time slot in response to different electricity prices can be induced by an auxiliary parameter denoted as $\rho^{j}$ and defined as:

$$
\rho^{j}=\frac{\left(\alpha^{j}-\alpha_{\min }\right)}{\left(\alpha_{\max }-\alpha_{\min }\right)} .
$$

Similar to Equation (8), a larger value preference term $\rho^{j}$ is obtained from Equation (9) when the electricity price $\alpha^{j}$ becomes higher and vice versa for $j=1, \ldots, J$. 


\subsection{Objective Function}

An optimal charging and discharging scheduling is proposed to coordinate the charging and discharging rates of connected EVs along with the power dispatches of ESS and power grid at every $j$-th time step based on the charging and discharging weight priorities (i.e., $w_{n}^{c, j}$ and $w_{n}^{d, j}$ ), as well as the preference on electricity prices (i.e., $\rho_{n}^{c, j}, \rho_{n}^{d, j}$, and $\rho^{j}$ ), without violating their respective constraints. Define $P_{E S S}^{c, \max }$ and $P_{n}^{d, \max }$ as the maximum charging and discharging powers of an ESS, respectively, while the associated charging and discharging rate ratios at any $j$-th time step are represented by two continuous variables, $s_{E S S}^{c, j} \in[0,1]$ and $s_{E S S}^{d, j} \in[0,1]$, respectively. The electricity purchased and sold at every $j$-th time step is denoted as $P_{\text {grid }}^{b, j}$ and $P_{\text {grid }}^{s, j}$, respectively. The objective function of scheduling optimization is then formulated to ensure that the satisfaction of EV owners in terms of completing all charging requests is maximized and the operational costs of the parking station are simultaneously minimized. To attain these contradictory goals, the objective function to be maximized at every time step is expressed as:

$$
\Phi^{j}=\sum_{j=k}^{J}\left(\sum_{n=1}^{N}\left(s_{n}^{c, j} P_{n}^{c, m a x} w_{n}^{c, j} \rho_{n}^{c, j}+s_{n}^{d, j} P_{n}^{d, m a x} w_{n}^{d, j} \rho_{n}^{d, j}\right)+\rho^{j}\left(s_{E S S}^{d, j} P_{E S S}^{d, m a x}-s_{E S S}^{c, j} P_{E S S}^{c, \text { max }}\right)+\rho^{j}\left(P_{\text {grid }}^{s, j}-P_{\text {grid }}^{b, j}\right)\right) .
$$

As shown in Equation (10), the parking station operator maximizes the charging power of connected EVs, especially during the time intervals with lower electricity prices, so that these EVs can be fully charged as soon as possible and the right of charging can be passed to more connected and yet uncharged EVs. The goal of maximizing the total charging rate of connected EVs at the current $k$-th time step is formulated as the product of charging priorities $w_{n}^{c, j}$, preference on electricity price for charging $\rho_{n}^{c, j}$, and maximum charging power $P_{n}^{c, m a x}$ evaluated from the current $k$-th time step to the end of day, i.e., $j=k, \ldots, J$ as mentioned in Equation (10).

The proposed EV charging and discharging scheduling aims to minimize the operational costs of the parking station by coordinating the V2G operation of EVs and the power dispatches of the power and ESS. As the electricity prices are high enough, the CMS is to discharge the energy stored in EVs' batteries and utilize the energy to charge other EVs or sell to the power grid. This strategy is formulated as the product of discharging priorities $w_{n}^{d, j}$, the preference on electricity price for discharging $\rho_{n}^{d, j}$, and the maximum discharging power $P_{n}^{d, m a x}$ evaluated from the current $k$-th time step to the end of the day, as shown in Equation (10). Apart from exploiting the V2G capability of connected EVs, the operational costs of the parking station can be further minimized by maximizing the net discharging power of ESS, denoted as $\left(s_{E S S}^{d, j} P_{E S S}^{d, \max }-s_{E S S}^{c, j} P_{E S S}^{c, \max }\right)$, and the net electricity sold to the grid, denoted as $\left(P_{\text {grid }}^{s, j}-P_{\text {grid }}^{b, j}\right)$, evaluated from the current $k$-th time step to the end of the day. The auxiliary parameter $\rho^{j}$ plays an essential role by inducing ESS discharging and electricity selling to the grid at time periods with higher electricity prices, while both ESS charging and electricity purchasing from the grid are preferred in the time periods with lower electricity prices.

\subsection{Optimization Constraints}

Define $P_{\text {grid }}^{b, j}$ and $P_{\text {grid }}^{s, j}$ as the amount of electricity purchased from and sold to the power grid at every $j$-th time step, respectively. The solar generation obtained from PVS at the same time period is denoted as $P_{P V}^{j}$. A demand-supply constraint is then defined to ensure the parking station load consisting of the total charging loads of EVs and ESS as well as the electricity to be sold to the grid at any $j$-th time step is balanced. Therefore,

$$
P_{\text {grid }}^{s, j}+s_{E S S}^{c, j} P_{E S S}^{c, \text { max }}+\sum_{n=1}^{N} s_{n}^{c, j} P_{n}^{c, \text { max }}=P_{\text {grid }}^{b, j}+P_{P V}^{j}+s_{E S S}^{d, j} P_{E S S}^{d, \max }+\sum_{n=1}^{N} s_{n}^{d, j} P_{n}^{d, \max }, j=k, \ldots, J .
$$


Note that $P_{P V}^{k}$ is measured at the current $k$-th time step while $P_{P V}^{j}$ for $j=(k+1), \ldots, J$ are based on an estimated profile that can be obtained using statistical modeling via the Markov model [40] or multi-step-ahead prediction with different neural network variants [41]. Both statistical modeling and multi-step-ahead prediction problems are beyond the scope of this work and hence will not be investigated further.

In order to prevent the overloading of the parking station, the total powers obtained from power grid $P_{\text {grid }}^{b, j}$ at any $j$-th time step must not exceed the associated capacity limit, $P_{\text {grid }}^{\text {max }, j}$. Similar restriction of $P_{\text {grid }}^{\max , j}$ is also imposed for the power discharged back to the grid $P_{g r i d}^{s, j} . P_{\text {grid }}^{\max , j}$ can either be a fixed or time-varying parameter, depending on whether a parking station operator participates in other demand response programs offered by the utility (e.g., load curtailment). While the market bidding mechanism used to clear the time-varying profile of $P_{\text {grid }}^{\text {max } j}$ in the day-ahead electricity market has drawn wide research interest [42-44], this research topic is beyond the scope of the current study and hence will not be further investigated. Furthermore, it is necessary to prohibit a scenario in which power discharge back to the grid and power drawn from the grid occur simultaneously in the parking station. To this end, both the capacity limit constraints of $P_{\text {grid }}^{b, j}$ and $P_{\text {grid }}^{s, j}$ as well as the aforementioned power exchange logic can be defined as:

$$
\begin{gathered}
P_{\text {grid }}^{b, j} \leq u_{\text {grid }}^{j} P_{\text {grid }}^{\text {max }, j}, j=k, \ldots, J, \\
P_{\text {grid }}^{s, j} \leq\left(1-u_{\text {grid }}^{j}\right) P_{\text {grid }}^{\text {max }, j}, j=k, \ldots, J,
\end{gathered}
$$

where $u_{\text {grid }}^{j} \in\{0,1\}$ is a binary auxiliary variable denoting whether the electricity needs to be drawn from or discharged back to grid at every $j$-th time step as shown below:

$$
u_{\text {grid }}^{j}=\left\{\begin{array}{l}
1, \text { if power is drawn from grid } \\
0, \text { otherwise }
\end{array} .\right.
$$

For each $n$-th charging pole connected with an EV, the initial SOC of EV is recorded as $\gamma_{n}^{\text {int }}$ upon its arrival time step of $\kappa_{n}^{\text {arr }}$, i.e.,

$$
\gamma_{n}^{j}=\gamma_{n}^{i n t}, \text { if } j=\kappa_{n}^{a r r}, n=1, \ldots, N
$$

The $n$-th connected EV can be charged, discharged, or remain idle at every $j$-th time step for $n=1, \ldots, N, j=\kappa_{n}^{a r r}, \ldots, \kappa_{n}^{d e p}$. During the scheduling process, the charging and discharging powers of each connected $\mathrm{EV}$ are regulated without violating its rated maximum charging and discharging powers (i.e., $P_{n}^{c, \max }$ and $P_{n}^{d, \max }$ ), respectively. A power exchange logic is further implemented to prevent the simultaneous charging and discharging of EV's battery at any scheduling period. Hence,

$$
\begin{gathered}
s_{n}^{c, j} P_{n}^{c, \text { max }} \leq u_{n}^{j} P_{n}^{c, \text { max }}, n=1, \ldots, N, j=\kappa_{n}^{a r r}, \ldots, \kappa_{n}^{\text {dep }}, \\
s_{n}^{d, j} P_{n}^{d, \max } \leq\left(1-u_{n}^{j}\right) P_{n}^{d, \max }, n=1, \ldots, N, j=\kappa_{n}^{a r r}, \ldots, \kappa_{n}^{\text {dep }},
\end{gathered}
$$

where $u_{n}^{j} \in\{0,1\}$ is a binary auxiliary variable introduced to indicate the charging and discharging status of each $n$-th connected EV at the $j$-th time step such that

$$
u_{n}^{j}=\left\{\begin{array}{l}
1, \text { if EV is charged } \\
0, \text { if EV is discharged }
\end{array} .\right.
$$


Notably, the idle state of the $n$-th connected EV can be described by Equations (16) or (17) because both decision variables referring to the charging and discharging rate ratios of EV (i.e., $s_{n}^{c, j}$ and $s_{n}^{d, j}$ ) are allowed to have zero values during its scheduling periods of $j=\kappa_{n}^{\text {arr }}, \ldots, \kappa_{n}^{\text {dep }}$.

The current SOC level $\gamma_{n}^{j}$ of every $n$-th connected EV at any $j$-th time step can be obtained by considering (a) the SOC of the previous slot $\gamma_{n}^{j-1}$; (b) the increment of SOC due to the energy provided to the EV's battery if it is charged; and (c) the decrement of SOC due to the energy released from the EV's battery if it is discharged. Let $\eta_{n}^{c}$ and $\eta_{n}^{d}$ be the charging and discharging efficiencies of the $n$-th connected EV. Given the sampling time $T_{s}$, the SOC of EV's battery at each time step is updated as follow:

$$
\gamma_{n}^{j}=\gamma_{n}^{j-1}+\frac{\eta_{n}^{c} P_{n}^{c, \max } S_{n}^{c, j} T_{s}}{E_{n}^{c a p}}-\frac{P_{n}^{d, \max } S_{n}^{d, j} T_{s}}{\eta_{n}^{d} E_{n}^{c a p}}, n=1, \ldots, N, j=\kappa_{n}^{a r r}, \ldots, \kappa_{n}^{d e p}
$$

At any $j$-th step, the SOC of each connected EV is restricted by an upper SOC limit $\gamma_{n}^{\max }$ and a lower SOC limit $\gamma_{n}^{\min }$ to avoid the overcharging and deep discharging of battery, respectively, i.e.,

$$
\begin{gathered}
\gamma_{n}^{j-1}+\frac{\eta_{n}^{c} P_{n}^{c, \max } s_{n}^{c, j} T_{s}}{E_{n}^{c a p}}-\frac{P_{n}^{d, \max } s_{n}^{d, j} T_{s}}{\eta_{n}^{d} E_{n}^{c a p}} \leq \gamma_{n}^{\max }, n=1, \ldots, N, j=\kappa_{n}^{a r r}, \ldots, \kappa_{n}^{d e p}, \\
\gamma_{n}^{j-1}+\frac{\eta_{n}^{c} P_{n}^{c, \max } s_{n}^{c, j} T_{s}}{E_{n}^{c a p}}-\frac{P_{n}^{d, \max } s_{n}^{d, j} T_{s}}{\eta_{n}^{d} E_{n}^{c a p}} \geq \gamma_{n}^{\min }, n=1, \ldots, N, j=\kappa_{n}^{a r r}, \ldots, \kappa_{n}^{d e p} .
\end{gathered}
$$

The charging of every EV needs to guarantee the minimum energy requirement for its next journey. For this reason, the SOC of EV connected to the $n$-th charging pole must satisfy the departure limit, denoted as $\gamma_{n}^{d e p}$, when it leaves the parking station during the time step $\kappa_{n}^{\text {dep }}$ as shown below:

$$
\gamma_{n}^{j-1}+\sum_{j=k}^{\kappa_{n}^{d e p}}\left(\frac{\eta_{n}^{c} P_{n}^{c, m a x} s_{n}^{c, j} T_{s}}{E_{n}^{c a p}}-\frac{P_{n}^{d, m a x} s_{n}^{d, j} T_{s}}{\eta_{n}^{d} E_{n}^{c a p}}\right) \geq \gamma_{n}^{d e p}, n=1, \ldots, N .
$$

The ESS is essentially similar to the EV's battery except that the former entity is available in the parking station throughout the scheduling process, and therefore offers less uncertain characteristics. At the beginning of each day, the initial SOC of ESS is recorded as $\gamma_{E S S}^{\text {int }}$, i.e.,

$$
\gamma_{E S S}^{j}=\gamma_{E S S}^{\text {int }}, \text { if } j=1
$$

Similar to the EV's battery, the scheduling of ESS is also decomposed into two variables, $s_{E S S}^{c j}$ and $s_{E S S}^{d, j}$, denoting the charging and discharging operations, respectively, for $j=1, \ldots, J$. Both the charging and discharging powers of ESS are controllable and yet constrained by their upper limits, given as $P_{E S S}^{c, \max }$ and $P_{E S S}^{d, \max }$, respectively. Furthermore, an ESS cannot be charged and discharged at the same time period. These charging and discharging power constraints of ESS are represented as:

$$
\begin{gathered}
s_{E S S}^{c, j} P_{E S S}^{c, \max } \leq u_{E S S}^{j} P_{E S S}^{c, \max }, j=1, \ldots, J, \\
s_{E S S}^{d, j} P_{E S S}^{d, \max } \leq\left(1-u_{E S S}^{j}\right) P_{E S S}^{d, \max }, j=1, \ldots, J,
\end{gathered}
$$

where $u_{E S S}^{j} \in\{0,1\}$ is a binary auxiliary variable associated with ESS at the $j$-th time step such that

$$
u_{E S S}^{j}=\left\{\begin{array}{l}
1, \text { if ESS is charged } \\
0, \text { if ESS is discharged }
\end{array} .\right.
$$


The SOC of ESS at any $j$-th time step, denoted as $\gamma_{E S S}^{j}$, is updated based on its SOC from previous time step $\gamma_{E S S}^{j-1}$ and the changes of SOC due to the power charged or discharged by ESS at that time step. Let $E_{E S S}^{c a p}$ be the battery capacity of ESS, while the power losses experienced by ESS during the charging and discharging are represented by the charging efficiency, $\eta_{E S S}^{\mathcal{C}}$, and discharging efficiency, $\eta_{E S S}^{d}$. At any $j$-th time step, the SOC of ESS is computed as:

$$
\gamma_{E S S}^{j}=\gamma_{E S S}^{j-1}+\frac{\eta_{E S S}^{c} P_{E S S}^{c, \max } s_{E S S}^{c, j} T_{S}}{E_{E S S}^{c a p}}-\frac{P_{E S S}^{d, \max } s_{E S S}^{d, j} T_{s}}{\eta_{E S S}^{d} E_{E S S}^{c a p}} j=1, \ldots, J
$$

During the charging and discharging, the SOC level of ESS at any time periods should not exceed its upper limit $\gamma_{E S S}^{\max }$ to prevent the battery from being overcharged. Furthermore, the SOC of ESS cannot be less than the lower limit, $\gamma_{E S S}^{\min }$, as provided by the manufacturer to ensure reasonable battery life-span. Hence,

$$
\begin{gathered}
\gamma_{E S S}^{j}+\frac{\eta_{E S S}^{c} P_{E S S}^{c, m a x} s_{E S S}^{c, j} T_{S}}{E_{E S S}^{c a p}}-\frac{P_{E S S}^{d, m a x} s_{E S S}^{d, j} T_{S}}{\eta_{E S S}^{d} E_{E S S}^{c a p}} \leq \gamma_{E S S}^{\max }, j=1, \ldots, J, \\
\gamma_{E S S}^{j}+\frac{\eta_{E S S}^{c} P_{E S S}^{c, m a x} s_{E S S}^{c, j} T_{S}}{E_{E S S}^{c a p}}-\frac{P_{E S S}^{d, \max } s_{E S S}^{d, j} T_{S}}{\eta_{E S S}^{d} E_{E S S}^{c a p}} \geq \gamma_{E S S}^{\min }, j=1, \ldots, J .
\end{gathered}
$$

Since the ESS serves as an energy source in the parking station, it must have a minimum amount of energy to provide charging for incoming EVs every day. In order to guarantee the minimum energy requirement for next-day usage, the SOC of ESS must satisfy a threshold limit $\gamma_{E S S}^{\text {thres }}$ at the last time step of the scheduling process. Therefore,

$$
\gamma_{E S S}^{j}+\sum_{j=k}^{J}\left(\frac{\eta_{E S S}^{c} P_{E S S}^{c, \max } s_{E S S}^{c, j} T_{S}}{E_{E S S}^{c a p}}-\frac{P_{E S S}^{d, \max } S_{E S S}^{d, j} T_{S}}{\eta_{E S S}^{d} E_{E S S}^{c a p}}\right) \geq \gamma_{E S S}^{\text {thres }} .
$$

\subsection{Scheduling Optimization}

Given the objective function and constraints defined in the previous subsections, the real-time optimal charging scheduling scheme for an EV parking station considering both PVS and ESS at every $j$-th time step is a maximization problem defined as follows:

$$
\begin{aligned}
& \max _{s_{n, j}^{c, j}, n=1, \ldots, N, j=k, \ldots, J} \Phi^{i}=\sum_{j=k}^{I}\left(\sum_{n=1}^{N}\left(s_{n}^{c, j} P_{n}^{c, m a x} w_{n}^{c, j} \rho_{n}^{c, j}+s_{n}^{d, j} P_{n}^{d, \max } w_{n}^{d, j} \rho_{n}^{d, j}\right)+\rho^{j}\left(s_{E S S}^{d, j} P_{E S S}^{d, \max }-s_{E S S}^{c, j} S_{E S S}^{c, m a x}\right)+\rho^{j}\left(P_{g r i d}^{s, j}-P_{g r i d}^{b, j}\right)\right), \\
& s_{n}^{d, j}, n=1, \ldots, N, j=k, \ldots, J \\
& u_{n}^{j}, n=1, \ldots, N, j=k, \ldots, J \\
& s_{E S S}^{c, j}, j=k, \ldots, J \\
& s_{E S S}, j=k, \ldots, J \\
& u_{E S S}^{j}, j=k, \ldots, J \\
& P_{\text {grid }}^{b, j}, j=k, \ldots, J \\
& P_{\text {grid }}^{\text {s,j }}, j=k, \ldots, J \\
& u_{\text {grid }}^{j}, j=k, \ldots, J
\end{aligned}
$$

subject to the constraints of Equations (11)-(13), (16), (17), (20)-(22), (24), (25), and (28)-(30).

The optimization of Equation (31) is a mixed-integer linear programming (MILP) problem because it consists of both continuous decision variables (i.e., $s_{n}^{c, j}, s_{n}^{d, j}, s_{E S S^{c, j}}, s_{E S S^{\prime},}, P_{\text {grid }}^{b, j}$ and $P_{\text {grid }}^{s, j}$ ) and binary auxiliary variables (i.e., $u_{n}^{j}, u_{E S S}^{j}$, and $u_{\text {grid }}^{j}$ ) to be optimized for $n=1, \ldots, N$ and $j=k, \ldots, J$. General MILP techniques such as the branch and bound algorithm [45] and the branch and cut algorithm [46], available in most available commercial optimization solvers, can be used to solve the EV charging optimization problem in Equation (31) effectively and efficiently. The proposed charging scheduling scheme repeatedly calculates the charging or discharging rate for each connected EV and ESS at 
every time step until the end of the scheduling task. The SOC levels of connected EV and ESS can be updated using Equations (19) and (27), respectively, as soon as their latest charging or discharging rates are obtained from Equation (31) at every time step. Note that although the EV connected to the $n$-th charging pole only stays at the parking station for the time steps $j \in\left[\kappa_{n}^{\text {arr }}, \kappa_{n}^{\text {dep }}\right]$, the charging scheduling optimization in Equation (31) can be made from the current $k$-th time step to the end of the day, i.e., $j \in[k, J]$ for convenient calculation due to the fact that $w_{n}^{c, j}=w_{n}^{d, j}=\rho_{n}^{c, j}=\rho_{n}^{d, j}=0$ for $\forall j \in\left[\kappa_{n}^{d e p}, J\right]$, as defined in Equations (5)-(8), respectively.

\section{Computer Simulation}

\subsection{Simulation Settings}

Extensive performance evaluations are conducted in this section by considering an EV parking station consisting of $N=200$ charging poles. The total available time period $H$ for charging scheduling is set as $30 \mathrm{~h}$, including the $24 \mathrm{~h}$ of a given day and the first $6 \mathrm{~h}$ of the next day, due to the different operational cycles of EVs and ESS. The sampling interval length used for calculating the optimal charging scheduling is set as $T_{S}=15 \mathrm{~min}$ or $0.25 \mathrm{~h}$, leading to the total time step of $J=H / T_{S}=120$. The capacity limit used in the parking station to restrict the electricity drawn from or discharged to the power grid at any $j$-th time step is given as $P_{\text {grid }}^{\max , j}=400 \mathrm{~kW}$. The day-ahead electricity prices used in this simulation are obtained from [47]. The solar radiation data for a typical summer day in Taiwan, measured by the National Taipei University of Technology (NTUT), are considered. The following parameter settings are considered for ESS. Each ESS unit is assumed to have a battery capacity of $E_{E S S}^{c a p}=100 \mathrm{kWh}$. The charging and discharging of ESS are constrained at the upper limits of $P_{E S S}^{c, \max }=P_{E S S}^{d, \max }=25 \mathrm{~kW}$, considering the efficiencies of $\eta_{E S S}^{c}=\eta_{E S S}^{c}=0.95$. The initial and threshold limit SOC levels of ESS are set as $\gamma_{E S S}^{\text {int }}=\gamma_{E S S}^{\text {thres }}=0.80$. Meanwhile, the upper and lower SOC limits of ESS are set as $\gamma_{E S S}^{\max }=0.99$ and $\gamma_{E S S}^{\min }=0.30$, respectively. Let $U$ be the number of ESS units used in the performance evaluation, set as $U=20$.

To simulate a more realistic scenario of parking station utilization, the presence of both regular and random EVs is assumed and the ratio is set at 5:5. Regular EVs refer to those with relatively fixed arrival and departure times due to their predictable parking station utilization habits. Both the arrival and departure times of regular EVs are generated using Gaussian models, in which the arrival times follow a normal distribution with mean $\mu_{\text {arv }}=6: 00 \mathrm{a}$.m. and standard deviation $\sigma_{\text {arv }}=60 \mathrm{~min}$. The departure times are modeled using a normal distribution with $\mu_{d e p}=6: 00$ p.m. and $\sigma_{d e p}=120 \mathrm{~min}$. The random-arrival EVs have more unpredictable habits of using the parking station because of their random staying time. These uncertain utilization characteristics can be simulated by uniformly distributing both the arrival and departure time along the scheduling period $j \in[1, J]$. Suppose that the total number of incoming EVs at a parking station over an entire day, i.e., for $j=1, \ldots, J$, is equal to $M$. As the charging of an EV is completed and it leaves the parking station, other EVs could reuse the charging pole. Hence, each charging pole can provide charging to more than one EV during a day. The total incoming EVs considered in the simulation are $M=\{100,200,300,400,500\}$. With the number of charging poles $N=200$, variations of number of EVs along the time steps $j=1, \ldots, J$ are simulated in Figure 2a-e. It is shown that all the charging poles are fully occupied for a certain time period when $M$ increases to 300 or above.

Some key parameters of EVs used in the simulations are given as follows. The EVs with four types of battery capacities, i.e., $8 \mathrm{kWh}, 17 \mathrm{kWh}, 18 \mathrm{kWh}$, and $48 \mathrm{kWh}$ are considered and their proportions are set as $20 \%, 30 \%, 30 \%$, and $20 \%$ of the $\mathrm{M} \mathrm{EVs}$, respectively. The maximum charging and discharging powers of these EVs are $1.6 \mathrm{~kW}, 3.4 \mathrm{~kW}, 3.6 \mathrm{~kW}$, and $9.6 \mathrm{~kW}$, respectively. The charging and discharging of these EVs are conducted with the charging and discharging efficiencies that are uniformly distributed between [0.90, 0.99]. The initial and departure SOC of each EV is uniformly distributed in the range of $[0.40,0.60]$ and $[0.90,0.95]$, respectively. Furthermore, the SOC level of these EVs are constrained by 
an upper limit and a lower limit that are uniformly distributed between [0.95, 0.99] and [0.30, 0.40], respectively. All simulations are made using MATLAB 2014a on a personal computer with Intel ${ }^{\circledR}$ Core i5-4570 CPU @ $3.40 \mathrm{GHz}$ and the proposed EV charging optimization problem is solved using CPLEX $12.1[48]$.

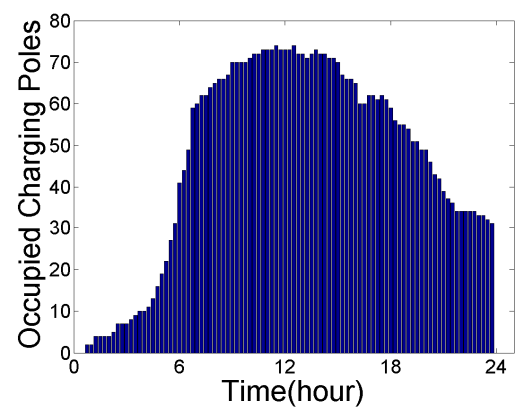

(a)

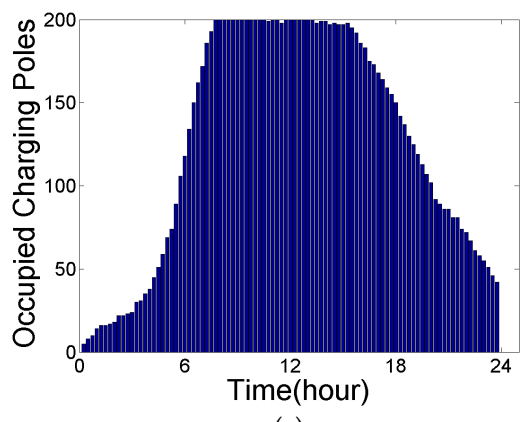

(c)

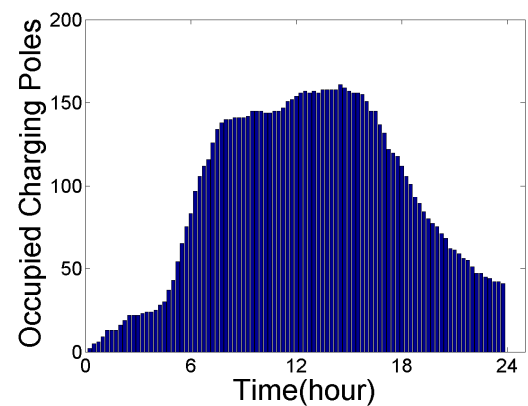

(b)

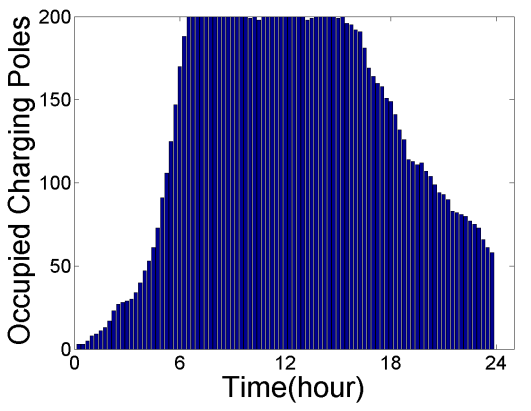

(d)

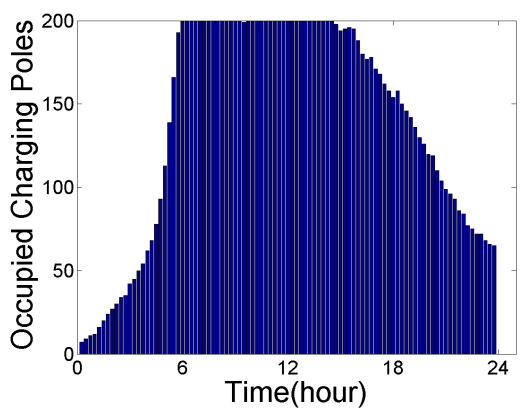

(e)

Figure 2. Charging pole accommodation status for (a) $M=100$; (b) $M=200$; (c) $M=300$; (d) $M=400$; and (e) $M=500$.

\subsection{Simulation of Optimal Charging Scheduling and Power Dispatches}

The charging scheduling of $M=500 \mathrm{EVs}$ in a parking station consisting of $U=20$ ESS units is simulated to evaluate the performance of the proposed EV charging optimization scheme. Figure 3 demonstrates the charging and discharging profiles of all connected EVs and the power dispatch profiles for both the power grid and ESS in a parking station along with the solar energy generated by PVS and dynamic electricity tariffs at different time slots beginning from 0:00 a.m. of a typical day to 6:00 a.m. of the next day. The net amount of grid electricity purchased or sold at every $j$-th time step is given as $P_{\text {net }}^{j}=P_{\text {grid }}^{b, j}-P_{\text {grid }}^{s, j}$ for $j=1, \ldots, J$. Positive values of $P_{\text {net }}^{j}$ imply that electricity is bought by the CMS from the power grid, while negative $P_{\text {net }}^{j}$ means the excess energy of the parking station is sold to the grid. From Figure 3, it is noticed that the total power received by the parking station at every time slot is balanced by its power demand, as given in Equation (11). The purchase and selling of electricity from/to the grid do not occur simultaneously. Both $P_{\text {grid }}^{b, j}$ and $P_{\text {grid }}^{s, j}$ are constrained below 
the capacity limit of $P_{\text {grid }}^{\text {max,j }}$, as stated in Equations (12)-(14). Similarly, the ESS does not charge and discharge concurrently and charging and discharges rates are restricted, as in Equations (24)-(26).

For the time periods where no solar power is generated from PVS to charge EVs, some connected EVs are scheduled by CMS to perform $\mathrm{V} 2 \mathrm{G}$ and release their stored energy in order to meet a portion of the EV charging demands. The remaining charging requests are satisfied by the electricity purchased by the CMS from the power grid. Notably, a portion of the grid electricity purchased at these time steps is also used to charge the ESS for later use, as will be explained in the following. At certain time steps between 9:00 a.m. and 11:00 a.m., the scheduled charging demands of connected EVs exceed the grid's capacity. These tremendous charging demands are first satisfied by the CMS using the solar power generation and the energy stored in ESS, followed by the electricity drawn from the grid. Similar observations are made from 5:00 p.m. to 7:00 p.m., except that the V2G capability of some connected EVs is also exploited at these time steps to cover a portion of the scheduled charging requests. In these two scenarios, drawing power from the grid is considered the last resort for the CMS to compensate for the power deficits of EV charging with the goal of minimizing the total charging costs. More connected EVs are scheduled to discharge than are scheduled to charge at the time periods with expensive electricity prices. The ESS is also discharged by the CMS, driven by the same economic motivation. Coincidentally, solar power generation at the time periods with higher electricity prices is relatively abundant. The total power supply contributed by PVS, ESS, and V2G operation of EVs is first used to charge the connected EVs with higher charging priorities, followed by selling the surplus power to the power grid.

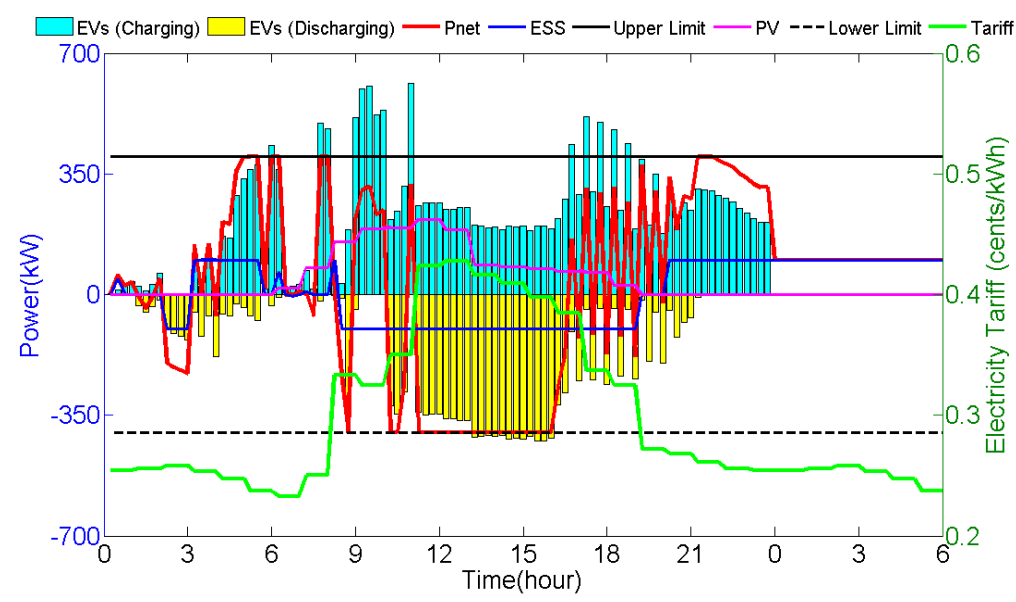

Figure 3. The variation of charging and discharging profiles of all connected EVs and the power dispatch profile for both the power grid and ESS in a parking station, along with the generation of solar power and a dynamic electricity tariff for $M=500$ and $U=20$.

Figure 4 presents the financial transactions recorded in a parking station for each time slot, including the revenue generated due to electricity selling, the overall charging cost incurred, and the net profit realized. Positive net profit implies that the surplus power available in a parking station is sold to the power grid, while negative net profit is equivalent to the net cost of purchasing electricity from the grid to compensate for the power deficit in the parking station. The cost optimization capability of the proposed approach is validated in Figure 4 because the majority of electricity purchases are shifted to the off-peak price periods, while most electricity sales are concentrated around the peak price periods. More connected EVs are scheduled for charging during the time periods when electricity tariffs are relatively low. The available power supplied by PVS, ESS, and V2G operation of EVs at these time periods is insufficient to meet the huge amount of charging load; therefore, the CMS needs to purchase electricity from the grid to compensate for the power deficits. For the scheduling periods with more expensive electricity prices, the CMS tends to discharge the energy stored in most connected 
EVs and ESS. Since the EV charging demands at these periods are relatively smaller, the CMS can sell the excess electricity to the power grid for more revenue.

The variations in average SOC for existing EVs and SOC of ESS in a parking station in response to the dynamic electricity tariff are illustrated in Figure 5. The average SOC of EVs starts to grow when the electricity tariff is relatively low. Similar behavior is demonstrated by the SOC profile of ESS. When the electricity price reaches its peak, the majority of connected EVs are scheduled by the CMS to discharge their stored energy to the grid, leading to the decrement of SOC. Meanwhile, the ESS is discharged for longer time intervals. The electricity released by ESS from 11:00 a.m. to 5:00 p.m. is sold to the grid, while these energy is used to cover a portion of EV charging demands for the remaining time periods. As shown in Figure 5, the variation in SOC profiles for both connected EVs and ESS is bounded by their respective lower and upper limits along the scheduling process, as stated by Equations (20) and (21) or (28) and (29), respectively. Finally, it is also observed that the average SOC of connected EVs and the SOC of ESS reach their respective threshold values before the end of the scheduling process, as specified by Equations (22) and (30).

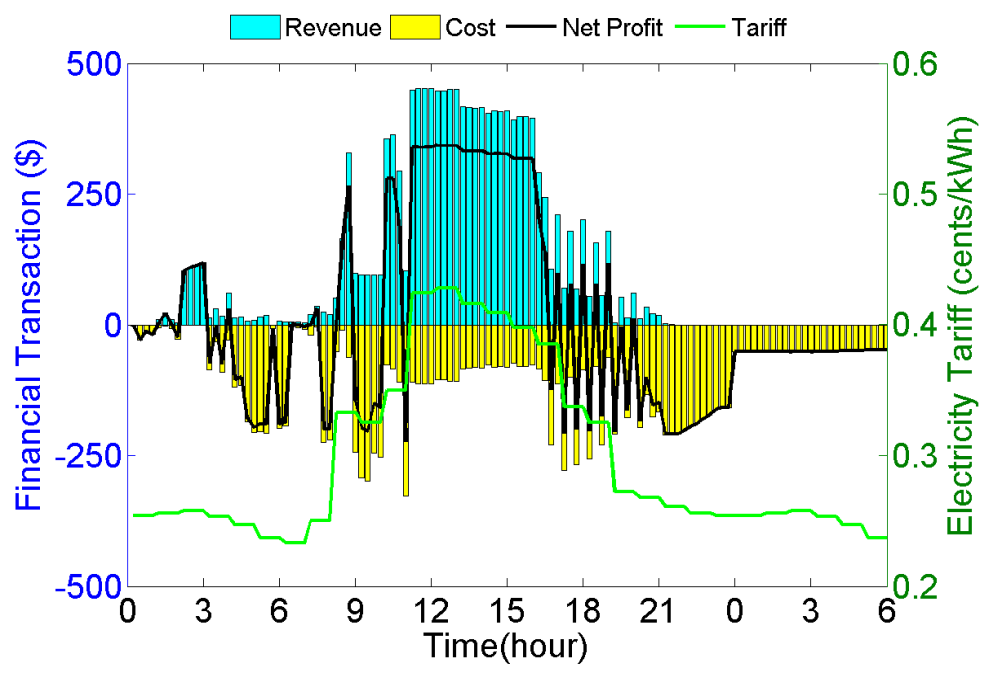

Figure 4. The variation of financial transactions recorded in a parking station, including the total revenue obtained due to electricity selling, total charging cost incurred, and total net profit realized in response to dynamic electricity tariff for $M=500$ and $U=20$.

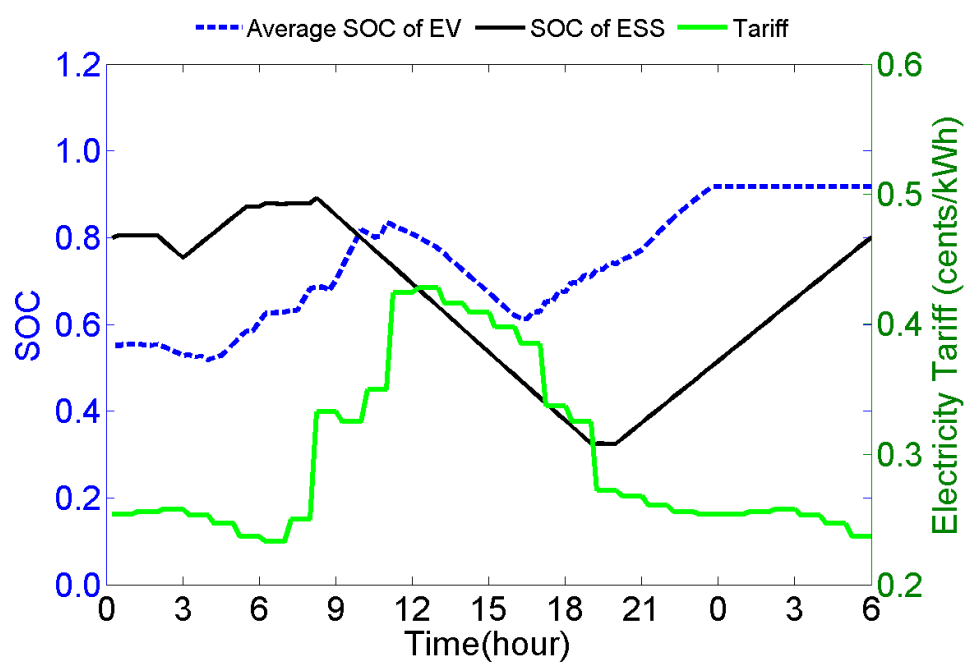

Figure 5. The variation of average SOC for existing EVs and SOC of ESS in a parking station in response to a dynamic electricity tariff for $M=500$ and $U=20$. 


\subsection{Charging Performance Comparisons}

The proposed optimal EV charging scheduling scheme is compared with a baseline method in order to verify its charging optimization capability. The baseline method is essentially similar to the proposed approach except that both PVS and ESS are discarded in the former method. Define $\gamma_{a v g}^{f}$ as the average SOC of all vehicles leaving the parking station, $\xi^{R}$ as the total revenue gained from electricity sales, $\xi^{C}$ as the total charging costs incurred, and $\xi^{P}$ as the net profit realized. Comparisons of $\gamma_{a v g}^{f}, \xi^{R}, \xi^{C}$, and $\xi^{P}$ between the baseline and proposed methods for $M=100$ to 500 are simulated and presented in Table 2.

Both the baseline and the proposed method produce similar values of $\gamma_{\text {avg }}^{f}$ for different values of $M$, implying that both methods are able to satisfy the charging requests of all EVs. On the contrary, the proposed approach greatly outperforms the baseline method from an economic point of view by producing lower charging costs $\xi^{C}$, higher revenue $\xi^{R}$, and higher net profit $\xi^{P}$ for $M=100$ to 500 . The negative values of $\xi^{P}$ produced by the baseline for different $M$ values imply that the revenue gained from electricity sales contributed by the V2G operation of EVs are insufficient to cover the total charging costs incurred, leading to the financial loss of the parking station. This challenge can be addressed by deploying both PVS and ESS in a parking station as alternative sources to provide EV charging. As demonstrated in Table 2, the proposed approach is able to achieve significant revenue and net profit gains by harnessing the economic value brought by both the PVS and ESS.

Table 2. Comparison of final average SOC of EVs $\gamma_{a v g}^{f}$, total revenue obtained $\xi^{R}$, total charging incurred $\xi^{C}$, and net profit gained $\xi^{P}$ for different number of EVs.

\begin{tabular}{cccccc}
\hline $\boldsymbol{M}$ & Method & $\gamma_{\text {avg }}^{f}$ & $\boldsymbol{\xi}^{R} \mathbf{( \$ )}$ & $\xi^{C} \mathbf{( \$ )}$ & $\boldsymbol{\xi}^{\boldsymbol{P}} \mathbf{( \$ )}$ \\
\hline \multirow{2}{*}{100} & Baseline & 0.919 & 1883.00 & 2517.55 & -634.55 \\
& Proposed & 0.919 & 5877.46 & 3975.75 & 1901.71 \\
\multirow{2}{*}{200} & Baseline & 0.916 & 3603.85 & 4815.94 & -1212.09 \\
& Proposed & 0.916 & 7572.46 & 6290.36 & 1282.10 \\
\multirow{2}{*}{300} & Baseline & 0.915 & 5219.75 & 6802.98 & -1583.23 \\
& Proposed & 0.915 & 8892.06 & 8057.68 & 834.38 \\
\multirow{2}{*}{400} & Baseline & 0.916 & 5243.41 & 6847.51 & -1604.10 \\
\multirow{2}{*}{500} & Proposed & 0.916 & 8777.38 & 8024.16 & 753.22 \\
& Baseline & 0.916 & 5657.87 & 7521.19 & -1863.32 \\
& Proposed & 0.916 & 8876.05 & 8396.65 & 479.40 \\
\hline
\end{tabular}

Notably, Table 2 also shows that the net profit realized, $\xi^{P}$, decreases with the number of EVs served despite the increase in $M$ implying that more electricity can potentially be contributed by the V2G operation of EVs. To justify these observations, the total revenue and charging costs contributed by different entities in the parking station (i.e., EVs, ESS, and PVS) are further analyzed in Figure 6. It is reported that the revenue and costs contributed by PVS and ESS are fixed with $M$ due to their constant parameter settings. While both the charging costs incurred and the revenue gained by EVs increases with the total number of vehicles, the former shows a more rapidly growing trend than the latter, resulting in the reduction of net profit gained with $M$. These observations can be justified by the different staying times of EVs at the parking station due to their different arrival and departure times. The majority of EVs, especially those with random arrival and departure times, are not able to sell their stored energy at the highest electricity price and perform charging at time periods with the lowest electricity price. Furthermore, for those EVs with a shorter staying time, the main priority is to provide sufficient energy to these vehicles in order to fulfill their charging needs instead of withdrawing their stored energy for electricity selling to the main grid. The uncertainties about EV charging demands due to their different or short staying times in parking stations becomes more notable as the number of vehicles further increases; these undesirable characteristics tend to offset the economic benefits brought by the V2G operation of EVs. 


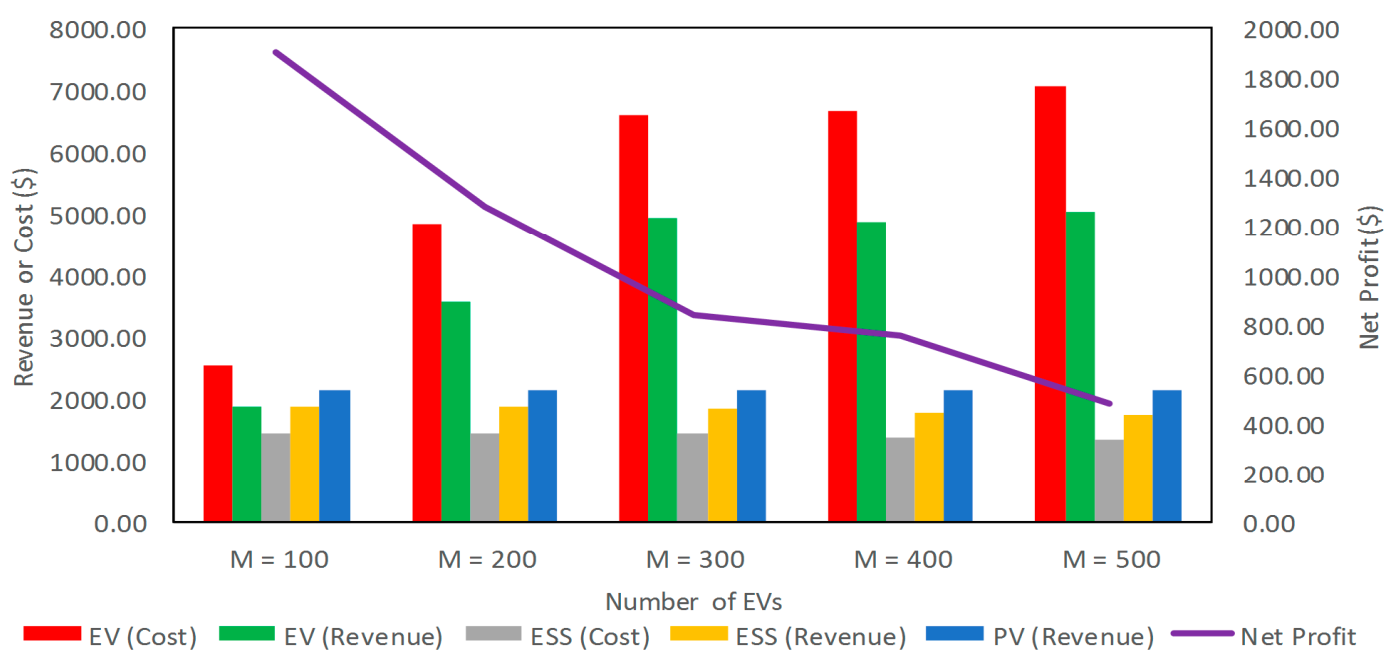

Figure 6. The variations of total revenue gained and charging costs incurred by different entities of a parking station (i.e., EVs, ESS, and PVS) with the change in the number of EVs served in the parking station for $U=20$ ESS units.

\section{Conclusions}

Solar energy has become a widely utilized renewable energy. This paper has integrated solar energy into energy resources for EV charging. In order to overcome the inevitable electricity intermittency of PVS, the EES is installed with PVS so that EES could supplement the necessary electricity during the period of electricity intermittency of PVS. The EV charging and discharging management thus becomes a complex optimization problem because not only does the charging and discharging of every EV in the parking need to be delicately scheduled, but the charging and discharging of ESS as well as the power dispatch of the grid and PVS also need to be optimally managed. A real-time optimal charging and discharging scheduling is formulated using MILP to coordinate the charging or discharging rates of EVs along with the power dispatches of the power grid and ESS, aiming to maximize EVs users' satisfaction in meeting charging and discharging requests while also maximizing the profit of the parking station. To ensure the proposed charging scheme is technically and financially feasible in parking stations, the constraints of the power grid, connected EV, and ESS are considered in the MILP optimization framework. Extensive simulations are conducted to evaluate the optimization capability of the proposed approach. The economic benefits of deploying both PVS and ESS in a parking station for EV charging are verified: this combination is able to generate more revenue and net profit from electricity sales than a scheme without PVS and ESS.

Some future works can be suggested based on the current proposal. First, the optimal charging and discharging scheduling in a parking station can be further extended by considering both price-based and incentive-based demand response programs in order to generate more revenue. Second, the ageing effect of ESS due to charging and discharging mechanisms can be considered during optimization in order to further preserve the lifetime of ESS by developing a suitable storage power loss model with linear characteristics that facilitates real-time optimization. To this end, a more comprehensive CMS framework can be developed by formulating the EV charging management in a parking station as a multi-objective optimization problem, aiming to minimize the operating costs of the EV parking station and the lifetime degradation of ESS simultaneously. Finally, the deployment of emerging energy sources such as a fuel cell system for EV charging in parking stations appears to be a promising research direction. More sophisticated optimization frameworks can be explored depending on the type of integration between the fuel cell system and the existing entities available at the parking station.

Acknowledgments: This work was supported by the Ministry of Science and Technology, Taiwan, R.O.C., under Grant MOST103-2221-E-027-050-MY3. 
Author Contributions: Leehter Yao substantially contributed to the supervision of this research. Zolboo Damiran substantially contributed to the algorithm design, the development of the algorithm, production and analysis of the results, and preparation of the manuscript for this study. Wei Hong Lim substantially contributed to the examination and interpretation of the results, and the review and proofreading of the manuscript.

Conflicts of Interest: The authors declare no conflict of interest.

\section{References}

1. Qian, K.; Zhou, C.; Allan, M.; Yuan, Y. Modeling of load demand due to EV battery charging in distribution systems. IEEE Trans. Power Syst. 2011, 26, 802-810. [CrossRef]

2. Su, W.; Eichi, H.; Zeng, W.; Chow, M.Y. A survey on the electrification of transportation in a smart grid environment. IEEE Trans. Ind. Inform. 2012, 8, 1-10. [CrossRef]

3. Mukherjee, J.C.; Gupta, A. A review of charge scheduling of electric vehicles in smart grid. IEEE Syst. J. 2014, 9, 1541-1553. [CrossRef]

4. Tie, S.F.; Tan, C.W. A review of energy sources and energy management system in electric vehicles. Renew. Sustain. Energy Rev. 2013, 20, 82-102. [CrossRef]

5. Shao, S.; Pipattanasomporn, M.; Rahman, S. Grid integration of electric vehicles and demand response with customer choice. IEEE Trans. Smart Grid 2012, 3, 543-550. [CrossRef]

6. Sortomme, E.; El-Sharkawi, M.A. Optimal charging strategies for unidirectional vehicle-to-grid. IEEE Trans. Smart Grid 2011, 2, 131-138. [CrossRef]

7. Ansari, M.; Al-Awami, A.T.; Sortomme, E.; Abidoeric, M.A. Coordinated bidding of ancillary services for vehicle-to-grid using fuzzy optimization. IEEE Trans. Smart Grid 2015, 6, 261-270. [CrossRef]

8. Saber, A.Y.; Venayagamoorthy, G.K. Efficient utilization of renewable energy sources by gridable vehicles in cyber-physical energy systems. IEEE Syst. J. 2010, 4, 285-294. [CrossRef]

9. Birnie, D. Solar-to-vehicle (S2V) system for powering commuters of the future. J. Power Sources 2009, 186, 539-542. [CrossRef]

10. Saber, A.Y.; Venayagamoorthy, G.K. Plug-in vehicles and renewable energy sources for cost and emission reductions. IEEE Trans. Ind. Electron. 2011, 58, 1229-1238. [CrossRef]

11. Jin, C.; Tang, J.; Ghosh, P. Optimizing electric vehicle charging: A customer's perspective. IEEE Trans. Veh. Technol. 2013, 62, 2919-2927. [CrossRef]

12. Xu, Z.; Hu, Z.; Song, Y.; Zhao, W.; Zhang, Y. Coordination of PEVs charging across multiple aggregators. Appl. Energy 2014, 136, 582-589. [CrossRef]

13. Jin, C.; Tang, J.; Ghosh, P. Optimizing electric vehicle charging with energy storage in the electricity market. IEEE Trans. Smart Grid 2013, 4, 311-320. [CrossRef]

14. Kuran, M.S.; Viana, A.C.; Iannone, L.; Kofman, D.; Mermoud, G.; Vasseur, J.P. A smart parking lot management system for scheduling the recharging of electric vehicles. IEEE Trans. Smart Grid 2015, 6, 2942-2953. [CrossRef]

15. Yao, L.; Lim, W.H.; Tsai, T.S. A real-time charging scheme for demand response in electric vehicle parking station. IEEE Trans. Smart Grid 2017, 8, 52-62. [CrossRef]

16. He, Y.; Venkatesh, B.; Guan, L. Optimal scheduling for charging and discharging of electric vehicles. IEEE Trans. Smart Grid 2012, 3, 1095-1105. [CrossRef]

17. Jian, L.; Zhu, X.; Shao, Z.; Niu, S.; Chan, C.C. A scenario of vehicle-to-grid implementation and its double-layer optimal charging strategy for minimizing load variance within regional smart grids. Energy Convers. Manag. 2014, 78, 508-517. [CrossRef]

18. Han, S.; Han, S.; Sezaki, K. Development of an optimal vehicle-to-grid aggregator for frequency regulation. IEEE Trans. Smart Grid 2010, 1, 65-72.

19. Su, W.; Chow, M.Y. Performance evaluation of an EDA-based large-scale plug-in hybrid electric vehicle charging algorithm. IEEE Trans. Smart Grid 2012, 3, 308-315. [CrossRef]

20. Su, W.; Chow, M.Y. Performance evaluation of a PHEV parking station using particle swarm optimization. In Proceedings of the 2011 IEEE Power Energy Society General Meeting, Detroit, MI, USA, 24-29 July 2011.

21. Su, W.; Chow, M.Y. Computational intelligence-based energy management for a large-scale PHEV / PEV enabled municipal parking deck. Appl. Energy 2012, 96, 171-182. [CrossRef]

22. Zhang, L.; Li, Y. A game-theoretic approach to optimal scheduling of parking-lot electric vehicle charging. IEEE Trans. Veh. Technol. 2016, 65, 4068-4078. [CrossRef] 
23. Moeini-Aghtaie, M.; Abbaspour, A.; Fotuhi-Firuzabad, M. Online multicriteria framework for charging management of PHEVs. IEEE Trans. Veh. Technol. 2014, 63, 3028-3037. [CrossRef]

24. Gao, S.; Chau, K.T.; Liu, C.; Wu, D.; Chan, C.C. Integrated energy management of plug-in electric vehicles in power grid with renewables. IEEE Trans. Veh. Technol. 2014, 63, 3019-3027. [CrossRef]

25. Jin, C.; Sheng, X.; Ghosh, P. Optimized electric vehicle charging with intermittent renewable energy sources. IEEE J. Sel. Top. Signal Process. 2014, 8, 1063-1072. [CrossRef]

26. Wang, R.; Xiao, G. Two-stage mechanism for massive electric vehicle charging involving renewable energy. IEEE Trans. Veh. Technol. 2016, 65, 4159-4171. [CrossRef]

27. Tushar, W.; Yuen, C.; Huang, S.; Smith, D.B.; Poor, H.V. Cost minimization of charging stations with photovoltaics: An approach with EV classification. IEEE Trans. Intell. Transp. Syst. 2016, 17, 156-169. [CrossRef]

28. Mohamed, A.; Salehi, V.; Ma, T.; Mohammed, O.A. Real-time energy management algorithm for plug-in hybrid electric vehicle charging parks involving sustainable energy. IEEE Trans. Sustain. Energy 2014, 5, 577-586. [CrossRef]

29. Nehrir, M.H.; Wang, C.; Strunz, K. A review of hybrid renewable/alternative energy systems for electric power generation: Configurations, control, and applications. IEEE Trans. Sustain. Energy 2011, 2, 392-403. [CrossRef]

30. Chen, Y.K.; Wu, Y.C.; Song, C.C.; Chen, Y.S. Design and implementation of energy management system with fuzzy control for DC microgrid systems. IEEE Trans. Power Electron. 2013, 28, 1563-1570. [CrossRef]

31. Lagorse, J.; Simoes, M.G.; Miraoui, A. A multiagent fuzzy-logic-based energy management of hybrid systems. IEEE Trans. Ind. Appl. 2009, 45, 2123-2129. [CrossRef]

32. Arcos-Aviles, D.; Pascual, J.; Marroyo, L.; Sanchis, P.; Guinjoan, F. Fuzzy logic-based energy management system design for residential grid-connected microgrids. IEEE Trans. Smart Grid 2016. [CrossRef]

33. Pascual, J.; Barricarte, J.; Sanchis, P.; Marroyo, L. Energy management strategy for a renewable-based residential microgrid with generation and demand forecasting. Appl. Energy 2015, 158, 12-25. [CrossRef]

34. Mohamed, A.; Mohammed, O. Real-time energy management scheme for hybrid renewable energy systems in smart grid applications. Electr. Power Syst. Res. 2013, 96, 133-143. [CrossRef]

35. Ren, H.; Wu, Q.; Gao, W.; Zhou, W. Optimal operation of a grid-connected hybrid PV/fuel cell/battery energy system for residential applications. Energy 2016, 113, 702-712. [CrossRef]

36. Erdinc, O.; Paterakis, N.G.; Mendes, T.D.P.; Bakirtzis, A.G. Smart household operation considering bi-directional EV and ESS utilization by real-time pricing-based DR. IEEE Trans. Smart Grid 2015, 6, 1281-1291. [CrossRef]

37. Riffonneau, Y.; Bacha, S.; Barruel, F.; Ploix, S. Optimal power flow management for grid connected PV systems with batteries. IEEE Trans. Sustain. Energy 2011, 2, 309-320. [CrossRef]

38. Wang, Y.; Lin, X.; Pedram, M. A near-optimal model-based control algorithm for households equipped with residential photovoltaic power generation and energy storage systems. IEEE Trans. Sustain. Energy 2016, 7, 77-86. [CrossRef]

39. Wang, D.; Ge, S.; Jia, H.; Wang, C.; Zhou, Y.; Lu, Y.; Kong, X. A demand response and battery storage coordination algorithm for providing microgrid tie-line smoothing services. IEEE Trans. Sustain. Energy 2014, 5, 476-486. [CrossRef]

40. Tushar, W.; Huang, S.; Yuen, C.; Zhang, J.; Smith, D.B. Synthetic generation of solar states for smart grid: A multiple segment Markov chain approach. In Proceedings of the 2014 IEEE PES Innovative Smart Grid Technologies Conference Europe (ISGT-Europe), Istanbul, Turkey, 12-15 October 2014.

41. Benmouiza, K.; Cheknane, A. Forecasting hourly global solar radiation using hybrid k-means and nonlinear autoregressive neural network models. Energy Convers. Manag. 2013, 75, 561-569. [CrossRef]

42. Nguyen, D.T.; Negnevitsky, M.; Groot, M.D. Walrasian market clearing for demand response exchange. IEEE Trans. Power Syst. 2012, 27, 535-544. [CrossRef]

43. Menniti, D.; Costanzo, F.; Scordino, N.; Sorrentino, N. Purchase-bidding strategies of an energy coalition with demand-response capabilities. IEEE Trans. Power Syst. 2009, 24, 1241-1255. [CrossRef]

44. Campos-Nanez, E.; Fabra, N.; Garcia, A. Dynamic auctions for on-demand services. IEEE Trans. Syst. Man Cybern. A Syst. Hum. 2007, 37, 878-886. [CrossRef]

45. Lawler, E.L.; Wood, D.E. Branch-and-bound methods: A survey. Oper. Res. 1966, 14, 699-719. [CrossRef]

46. Padberg, M.; Rinaldi, G. A branch-and-cut algorithm for the resolution of large-scale symmetric traveling salesman problems. SIAM Rev. 1991, 33, 60-100. [CrossRef] 
47. Réseau de Transport D'électricité. Available online: http://clients.rte-france.com/index_en.jsp (accessed on 21 October 2016).

48. IBM. ILOG CPLEX V12. 1: Users Manual for CPLEX; International Business Machines Corporation: New York, NY, USA, 2009; Volume 46, p. 157. article distributed under the terms and conditions of the Creative Commons Attribution (CC BY) license (http://creativecommons.org/licenses/by/4.0/). 